\title{
On the Safety Assessment for the Collision Risk Between the Civilian and Military Airplanes
}

\author{
Ma Jiacheng \\ Air Traffic Control and Navigation College, \\ Air Force Engineering University, \\ Xian, China \\ e-mail: 727032612@qq.com
}

\author{
Wang jianbo \\ Air Traffic Control and Navigation College, \\ Air Force Engineering University, \\ Xian, China \\ e-mail: jianbowang2008@163.com
}

\begin{abstract}
This paper is mainly aimed at making an assessment of the flying collision risks between the military and civilian flights. The collision risk model of military training airspace and civil flight route was constructed based on the study and research of the collision risk of civil flight Event model. The probability model of lateral position deviation of military aircraft was established according to the characteristics of military flight fact. The frequency of collision box traversing the spacer (GERh) in the Event model was recalculated based on the transverse position deviation probability model of the civil aircraft. A tactical action was selected to run a simulation and prove the rationality of military and civil safety distance interval standard. The corresponding safety distance intervals were determined by calculating the collision risk of different distance intervals. A few suggestions about the use of airspace were proposed according to the research of key points collision risk during the process of flight. These can provide some references for the more flexible use of the airspace. As the results indicate that the current safety distance interval standard $(10 \mathrm{~km})$ can't meet the required safety target level. And there is at least another $7 \mathrm{~km}$ distance need to be added to meet safety requirement of the whole flight process. Therefore, extra $17 \mathrm{~km}$ need to be added to the safety distance interval other than the maneuver airspace when designing the training airspace. According to the collision risk degree of each key point during the flight process, the starting point of maneuver should be set near one side of air-route. Because the collision probability of same direction flights is smaller than that of reverse direction flights, aircrafts should be in the same direction to reduce the collision risk of the flight process .
\end{abstract}

Keywords-component; collision risk; safety assessment; collision box; interval

\section{INTRODUCTION}

With the application of performance-based navigation (PBN) technology, a new route structure and airspace interval.Civil aviation industry has entered a sustained and rapid development period, with all aspects of the transport volume increasing significantly. In particular, in terms of navigation, airspace users and aviation activities tend to be more diversified. At the same time, the transformation of military training continues to advance, the proportion of military training time and tactical training continues to improve. As a result, the demand for airspace resources has increased year by year. According to different training subjects, it is important to determine the safety interval between military and civilian flights and define the corresponding training airspace under the requirements of the target safety level, which is of great significance to improve the utilization rate of airspace and alleviate airspace conflict between civilian and military flights. In 1966, Reich[1] first proposed the REICH Collision Risk Model for the analysis of the safety of the air traffic route structure. In 2003, Brooker[2] proposed the Event Model to assess the risk of lateral collision. In 2008, Xu Xiaowao and some scientists[3-4] used the Event Model to evaluate the safety intervals in different directions of the route. The model was improved and the cylinder was used instead of the original rectangular box to improve the accuracy of the risk assessment. In 2010, Zhang Zhaoning etc.[5] conducted a safety assessment of the vertical separation based on Communication, Navigation and Surveillance (CNS). With the development of various risk models of collision, many scholars at home and abroad have carried on the thorough researches, and put forward various improvement models[6-7]. In 2014, Zhang Xiaoyan etc[8] improved the calculation method of lateral overlap probability, which was closer to the actual running condition, and evaluated the collision risk of Lhasa parallel route. In 2015, Cao Xingwu etc.[9] used ellipsoidal collision template instead of the traditional rectangular collision template to recalculate the risk of cross-road collision, and the test shows that this method is more accurate and reliable. The pairing approach to the close parallel runway can effectively improve the airport capacity. However, the above study is only for the collision interval between civil aircraft, for the safety interval between civil and military aviation of little literature discussion. According to the "Flight Interval", the safety interval between training airspace and route boundary is $10 \mathrm{~km}$, but it is not known whether it is suitable for all training subjects. In this paper, we construct the calculation model of civil and military collision risk by improving the Event model, and verify the rationality of the $10 \mathrm{~km}$ safety interval standard of civil and military aviation through a certain training subject and further determine the safety interval. 


\section{Civil AND MiLitARY AVIATION EVEnT MODEL}

The original Event model is a collision risk model for civil aviation routes and can not be directly used for the calculation of collision risk between civil and military aviation. The frequency at which the interval is lost per hour (GERh) in the original model is obtained through statistical data, but there is no statistical data between civil and military aviation. Therefore, according to the characteristics of civil and military aviation, the collision risk calculation model is needed.

The size of the rectangular collision box in the original model is redefined, and its length, width, height $\left(l_{\mathrm{hx}}, \mathrm{l}_{\mathrm{hy}}, \mathrm{l}_{\mathrm{hz}}\right)$ is calculated as follows:

$$
\left\{\begin{array}{l}
l_{\mathrm{h} x}=l_{\mathrm{m} x}+l_{\mathrm{j} x} \\
l_{\mathrm{h} y}=l_{\mathrm{m} y}+l_{\mathrm{j} y} \\
l_{\mathrm{hz}}=l_{\mathrm{mz}}+l_{\mathrm{j} z}
\end{array}\right.
$$

$l_{\mathrm{mx}}, \mathrm{l}_{\mathrm{my}}, \mathrm{l}_{\mathrm{mz}}$ and $\mathrm{l}_{\mathrm{jx}}, \mathrm{l}_{\mathrm{jy}}, \mathrm{l}_{\mathrm{jz}}$ are civil and military airplanes fuselage length, wingspan and fuselage height. In this way, the civil airplane can be seen as a point $\mathrm{B}$. When the point $\mathrm{B}$ and collision box A contact, we can think that airplane A, airplane B have a collision.

In the original model, each of the two airplanes will be regarded as a pair, while in this model, each military airplane and civilian airplane can be regarded as a pair. Since the two planes are either co-directional or reverse flight, the Event model is rewritten as:

$$
\begin{aligned}
& N_{\text {ay }}=\operatorname{GERh} \frac{2 E(\mathrm{~S})}{2 L}\left(l_{\mathrm{h} x}+\frac{U_{\mathrm{s}} l_{\mathrm{hy}}}{V_{\mathrm{S}}}\right) P_{z}(0)\left(1+\frac{W_{\mathrm{s}} l_{\mathrm{hy}}}{V_{\mathrm{S}} l_{\mathrm{hz}}}\right) \beta+ \\
& \operatorname{GERh} \frac{2 E(\mathrm{O})}{2 L}\left(l_{\mathrm{hx}}+\frac{U_{\mathrm{o}} l_{\mathrm{hy}}}{V_{\mathrm{O}}}\right) P_{z}(0)\left(1+\frac{W_{\mathrm{O}} l_{\mathrm{hy}}}{V_{\mathrm{O}} l_{\mathrm{hz}}}\right) b
\end{aligned}
$$

Nay is the risk of collision between the two planes on the parallel route of the same height; $\beta$ is the frequency of the average hourly flight of the military airplanes in the military airspace training airspac; $2 \mathrm{E}(\mathrm{S})$ is the number of civil airplanes in the same direction with the military airplanes within 2L,2E $(\mathrm{O})$ is the number of civil airplanes in the reverse direction with the military airplanes within $2 \mathrm{~L} ; \mathrm{U}_{\mathrm{S}}, \mathrm{V}_{\mathrm{S}}, \mathrm{W}_{\mathrm{S}}$ is the relative velocity in the longitudinal, lateral and vertical directions when airplane $A$ in the same flight flies through airplane B's spacer; $U_{O}, V_{O}$, and $W_{O}$ is the relative velocity of the two planes in the longitudinal, lateral and vertical directions when plane $\mathrm{A}$ in the reverse flight crosses the spacer of the plane B; assuming that $\mathrm{V}_{\mathrm{S}}=\mathrm{V}_{\mathrm{O}}, \mathrm{W}_{\mathrm{S}}=\mathrm{W}_{\mathrm{O}} ; \mathrm{Pz}(0)$ is the probability of vertical overlap between two planes at the same height.

\section{CALCULATION OF LATERAL OVERLAP PROBABILITY OF CIVIL AND MILITARY AIRPLANES}

\section{A. Reference coordinate system}

Horizontal "8" motor can be regarded as two turning maneuvers with same turning angle's radius in the horizontal flight. Take the turning center of the civil aviation route as the coordinate center; over the dots and perpendicular to the route center line to the route side as the x-axis. Route boundary and training airspace boundary is $10 \mathrm{~km}$ safety interval specified in the regulations, Schematic diagram of military and civil aviation maneuver is shown in Figure 1.

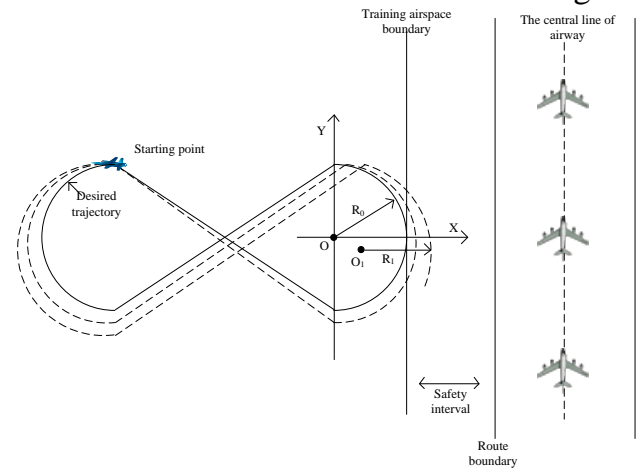

Fig.1 Schematic diagram of military and civil aviation maneuver

\section{B. The lateral position deviation of civil aviation}

According to the literature [7], the lateral error of the civil aircraft is generally in accordance with the probability distribution function fnorm_y ( $\mathrm{y}$ '), $\mathrm{y}$ is the distance of route aircraft away from the route center line ( $\mathrm{y}$ is the expected value, $\mathrm{y}^{\prime}$ is the actual value), which is 


$$
f_{\text {norm } \_y}\left(y^{\prime}\right)=\frac{1}{2 \sigma_{y}} \exp \left(-\left|\frac{R_{0}+20-y}{\sigma_{y}}\right|\right)
$$

$\sigma \mathrm{y}$ is the parameter corresponding to the general yaw error probability density function, which can be determined by the required navigation performance (RNP). The RNP directs the flight accuracy, which is the distance (nautical miles) where airplane on the route departs from the route centerline at $95 \%$ probability. According to the RNP, the parameter $\sigma_{\mathrm{y}}$ under the corresponding navigation condition can be calculated. According to the literature[5], when RNP is $4, \sigma_{\mathrm{y}}$ is 1.33 nautical miles.

\section{The lateral position deviation probabilistic model of military aviation}

As the military flight training track changes a lot, it cannot be estimated as civil aviation route position deviation. Therefore, the lateral position deviation of military aircraft can only be analyzed by the mathematical model, combined with flight characteristics. Taking the "8" flight as an example, the lateral error is determined by calculating the radius of the turning flight near the route and the change of its center.

Calculation of turning radius

Usually the fighter pilots' action errors obey the normal distribution, and their distribution functions are as follows:

$$
g\left(M_{\text {last }}\right)=\frac{1}{\sqrt{2 \pi} \sigma_{\mathrm{p}}} \exp \left(-\frac{\left(M_{\text {last }}-M_{\text {shlould }}\right)^{2}}{2 \sigma_{\mathrm{p}}^{2}}\right)
$$

$\mathrm{M}_{\text {shlould }}$ is expectation action, $\mathrm{M}_{\text {last }}$ is actual action, $\sigma_{\mathrm{p}}$ is standard deviation.

In the process of aircraft training, the factors that affect the maneuvering trajectory of the aircraft are mainly starting point, turning slope, turning speed, wind and the accuracy of its navigation facilities. As shown in Fig. 2, the desired maneuvering trajectory of the aircraft is shown by a solid line. Due to the effects of omnidirectional winds and actions, the trajectories of the aircraft are shown by dashed lines.

$$
R_{0}=\frac{V^{2}}{g \tan \gamma}
$$

This paper assumes that the expected flight speed of the aircraft is $\mathrm{V}=250 \mathrm{~m} / \mathrm{s}(900 \mathrm{~km} / \mathrm{h})$ and the expected slope is $\gamma=$ $45^{\circ}$. Due to the operator's motion error, it is subject to the normal distribution of equation (5). Under the influence of speed error and turning slope error, the turning radius is:

$$
R_{1}=\frac{\left(V^{\prime}\right)^{2}}{g \tan \left(\gamma^{\prime}\right)}
$$

The actual speed and the actual turning gradient are subject to the following normal distribution.

$$
\begin{aligned}
& f_{\gamma}\left(\gamma^{\prime}\right)=\frac{1}{\sqrt{2 \pi} \sigma_{\gamma}} \exp \left(-\frac{\left(45-\gamma^{\prime}\right)^{2}}{2 \sigma_{\gamma}^{2}}\right) \\
& f_{V}\left(V^{\prime}\right)=\frac{1}{\sqrt{2 \pi} \sigma_{V}} \exp \left(-\frac{\left(250-V^{\prime}\right)^{2}}{2 \sigma_{V}^{2}}\right)
\end{aligned}
$$

Calculation of turning track center

The actual centerline position on the side of the route is defined as $\mathrm{O}_{1}\left(\mathrm{x}_{1}, \mathrm{y}_{1}\right)$, and the desired center is defined as are $\mathrm{O}$ $\left(\mathrm{x}_{\mathrm{s}}, \mathrm{y}_{\mathrm{s}}\right)$, and only the influence of the turning process is taken into account in the process of maneuver. The actual center coordinates are:

$$
\left\{\begin{array}{l}
x_{1}=x_{\mathrm{s}}+R_{1} \cos \theta \\
y_{1}=y_{\mathrm{s}}-R_{1} \sin \theta
\end{array}\right.
$$

$\theta$ is the heading angle when the airplane begins to circulate. Assuming the circling heading deviation is only caused by the navigation accuracy, the airborne navigation device error, the pilots' operating errors, and the change of angle obeys the normal distribution.

$$
f_{\theta}\left(\theta^{\prime}\right)=\frac{1}{\sqrt{2 \pi} \sigma_{\theta}} \exp \left(-\frac{\left(\vartheta-\theta^{\prime}\right)^{2}}{2 \sigma_{\theta}^{2}}\right)
$$

$\theta^{\prime}$ is the actual heading angle and is the heading angle in the ideal state. According to the conservative values given in ICAO 8168 , the standard deviation $\sigma_{\theta}=2.6^{\circ}(0.045 \mathrm{rad})$, and the heading angle equation in the ideal state is: 


$$
\vartheta=\left\{\begin{array}{cc}
\pi-\arctan \left(\frac{y_{\mathrm{s}}}{x_{\mathrm{s}}}\right) x_{\mathrm{s}}>0, y_{\mathrm{s}} \neq 0 ! \\
2 \pi-\arctan \left(\frac{y_{\mathrm{s}}}{x_{\mathrm{s}}}\right) z_{\mathrm{s}}<0, y_{\mathrm{s}} \neq 0 \\
\frac{\pi}{2} \quad x_{\mathrm{s}}=0, y_{\mathrm{s}}=R_{0} \\
-\frac{\pi}{2} ? \quad x_{\mathrm{s}}=0, y_{\mathrm{s}}=-R_{0} \\
\pi \quad x_{\mathrm{s}}=R_{0}, y_{\mathrm{s}}=0 \\
0 \quad x_{\mathrm{s}}=-R_{0}, y_{\mathrm{s}}=0
\end{array}\right.
$$

\section{SIMULATION OF LATERAL DEVIATION OF MILITARY AND CIVIL AVIATION}

In the process of assessing the deviation of the maneuvering action, the evaluation is made by selecting different starting points because the lateral position deviation is affected by the starting points. This paper mainly studies the influence of the turning process, so 8 points in the turning process are selected as the starting point of the action. The starting points are shown in Figure 2.

There is a nonlinear term in the lateral deviation equation of military and civil aviation, and it is difficult to obtain the analytic expression of its distribution probability density function, so the numerical simulation method is adopted. According to the relevant civil aviation data, assuming that 24 fighters is protected in the military airports, and each fighter has $200 \mathrm{~h}$ training mission, five training airspace is under the jurisdiction of the airport, each time the time of activities in the airspace is a half of the total time length of the flight, the average frequency of training flight per hour in each training airspace is:

$$
\beta=\frac{24 \times 200}{365 \times 24} \times \frac{1}{2} \times \frac{1}{5}=0.054
$$

The other parameters of the collision risk simulation calculation are shown in Table I. Among them, the size of civil and military aircraft is public data of F16 and A380 passenger aircraft [6]. According to China's national conditions, the vertical safety interval is our radar control $10 \mathrm{~km}$ safety interval, rather than $120 \mathrm{~nm}$ in the literature [7]. The Monte Carlo method is used to select the random number of the probability density function distribution of velocity and slope, and the flight simulation of $n=1000000$ military and civil aircraft is carried out. The lateral deviation results are shown in Fig. 3 and Fig.4:

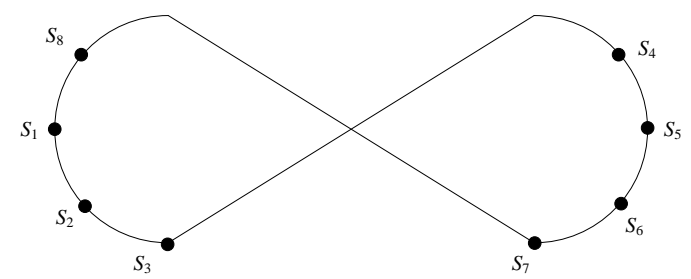

Fig.2 Selection of Starting Point

Table I Parameters of collision risk

\begin{tabular}{|llll|}
\hline Parameters & Values & Parameters & Values \\
\hline$E(\mathrm{~S})$ & 0.61 & $l_{\mathrm{mz}} / \mathrm{m}$ & 24.1 \\
$E(\mathrm{O})$ & 0.01 & $l_{\mathrm{j} x} / \mathrm{m}$ & 15.6 \\
$V /\left(\mathrm{m} \cdot \mathrm{s}^{-1}\right)$ & 250 & $l_{\mathrm{i} y} / \mathrm{m}$ & 9.45 \\
$\gamma$ & 45 & $l_{\mathrm{iz}} / \mathrm{m}$ & 5.09 \\
$\sigma_{V}$ & 15 & $U_{\mathrm{S}} /\left(\mathrm{m} \cdot \mathrm{s}^{-1}\right)$ & 3 \\
$P_{z}(0)$ & 0.5 & $V_{\mathrm{S}}$ or $V_{\mathrm{O}} /\left(\mathrm{m} \cdot \mathrm{s}^{-1}\right)$ & 5.23 \\
$L / \mathrm{km}$ & 10 & $W_{\mathrm{S}}$ or $W_{\mathrm{O}} /\left(\mathrm{m} \cdot \mathrm{s}^{-1}\right)$ & 0.58 \\
$l_{\mathrm{m} /} / \mathrm{m}$ & 72.8 & $\beta$ & 0.054 \\
$l_{\mathrm{m} \gamma} / \mathrm{m}$ & 79.8 & & \\
\hline
\end{tabular}

The lateral deviation of the civil aviation and the deviation of the military aviation are calculated subtraction according to the number of simulation times. Calculate the ratio between the difference less than 0 and the total number of simulations, and in the situation the frequency of the lateral interval loss of civil and military aviation GERh $=8.38 \times 10-5$. When the civil aircraft in the hover training is in the reverse flight at the same height, which is $E(S)=0.01$ and $E(O)=0.61$, GERh and the 
above parameters are brought into Eq. (2), $\mathrm{N}_{\text {ay_ same }}=2.58 \times 10^{-8}$ (times $\cdot$ Flight hour ${ }^{-1}$ ), $\mathrm{N}_{\text {ay_reverse }}=2.60 \times 10^{-8}$ (times $\bullet$ Flight hour $^{-1}$ ), where the total collision probability is $\mathrm{N}_{\text {total }}=5.18 \times 10^{-8}$ (times $\cdot$ Flight hour $\left.{ }^{-1}\right)$. Respectively, the calculation of 8 different starting points collision probability in the same and reverse direction is in Table II.

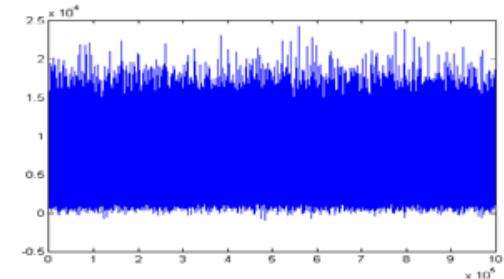

Fig.3 lateral deviation of military aviation

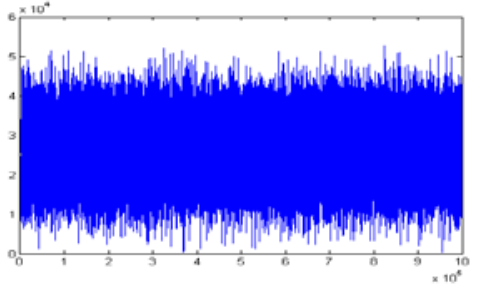

Fig.4 lateral deviation of civil aviation

TABLE II MILITARY AND CIVIL AVIATION COLLISION PROBABILITY AT DIFFERENT STARTING POINTS

\begin{tabular}{|ccccc|}
\hline $\begin{array}{r}\text { starting } \\
\text { point }\end{array}$ & GERh & $\mathrm{N}_{\text {ay_same }}$ & $\mathrm{N}_{\text {ay_reverse }}$ & $\mathrm{N}_{\text {total }}$ \\
\hline S1 & $9.56 \times 10^{-5}$ & $2.94 \times 10^{-8}$ & $2.96 \times 10^{-8}$ & $5.90 \times 10^{-8}$ \\
S2 & $8.38 \times 10^{-5}$ & $2.58 \times 10^{-8}$ & $2.60 \times 10^{-8}$ & $5.18 \times 10^{-8}$ \\
S3 & $7.30 \times 10^{-5}$ & $2.25 \times 10^{-8}$ & $2.26 \times 10^{-8}$ & $4.51 \times 10^{-8}$ \\
S4 & $6.34 \times 10^{-5}$ & $1.94 \times 10^{-8}$ & $1.95 \times 10^{-8}$ & $3.90 \times 10^{-8}$ \\
S5 & $5.45 \times 10^{-5}$ & $1.68 \times 10^{-8}$ & $1.69 \times 10^{-8}$ & $3.37 \times 10^{-8}$ \\
S6 & $1.05 \times 10^{-4}$ & $3.22 \times 10^{-8}$ & $3.24 \times 10^{-8}$ & $6.45 \times 10^{-8}$ \\
S7 & $1.25 \times 10^{-4}$ & $3.83 \times 10^{-8}$ & $3.86 \times 10^{-8}$ & $7.69 \times 10^{-8}$ \\
S8 & $1.14 \times 10^{-4}$ & $3.51 \times 10^{-8}$ & $3.53 \times 10^{-8}$ & $7.05 \times 10^{-8}$ \\
\hline
\end{tabular}

When the starting point is from the S1 point to the S5 point, the collision probability is gradually reducing, mainly because when the military aircraft moves along the anti-clockwise, it arrived on the side of the route in a very short time, thus omnidirectional wind has little impact. However, the impact of the pilot operation error mainly lead to the trajectory more biased to the other side of the extension line of the starting point in the track circle with the center line. The closer the turning point is to the route, the more difficult it is for the military aircraft to deviate from the training airspace due to the operator's operational error.

When the hover starting point is from the S5 point to the S7 point, the collision probability is gradually increasing, which is mainly affected by the pilot operating error. S3 and S7 are affected by the pilot operating error of the same, but from the S7 point began to maneuver to the side of the route for a long time, by the full impact of the wind, so the collision probability is high. As the aircraft continue to fly, the probability of collision began to gradually reduce.

For the same hover starting point, when hovering to the nearest route, the probability of collision of the military and civil aviation aircraft in the same direction is lower than the reverse flight. When the starting point is at the S5 point, the collision probability is the smallest; when the starting maneuvering point is at the S7 point, the collision probability is the largest. This is because the collision probability is affected by the pilot operating error and the omnidirectional wind. The S5 point is almost unaffected by the omnidirectional wind, and the S7 point is most affected by the above two. However, it is not difficult to find that the collision probability of individual points is greater than $5 \times 10^{-9}$. Obviously, there is a certain risk of collision setting the airspace boundary based on track boundary. Therefore, the collision probability under various safety margins is calculated. When the boundary distance between the ideal track boundary and the space boundary is $7 \mathrm{~km}$, the collision risk can meet the target safety level, see Table III'

TABLE III MILITARY AND CIVIL AVIATION COLLISION PROBABILITY AFTER INCREASED SAFETY DISTANCE INTERVAL

\begin{tabular}{|ccccc|}
\hline $\begin{array}{r}\text { starting } \\
\text { point }\end{array}$ & GERh & $\mathrm{N}_{\text {ay_same }}$ & $\mathrm{N}_{\text {ay_reverse }}$ & $\mathrm{N}_{\text {total }}$ \\
\hline S1 & $4.80 \times 10^{-6}$ & $1.48 \times 10^{-9}$ & $1.49 \times 10^{-9}$ & $2.96 \times 10^{-9}$ \\
S2 & $5.50 \times 10^{-6}$ & $1.69 \times 10^{-9}$ & $1.70 \times 10^{-9}$ & $3.40 \times 10^{-9}$ \\
S3 & $5.30 \times 10^{-6}$ & $1.63 \times 10^{-9}$ & $1.64 \times 10^{-9}$ & $3.27 \times 10^{-9}$ \\
S4 & $4.70 \times 10^{-6}$ & $1.44 \times 10^{-9}$ & $1.45 \times 10^{-9}$ & $2.89 \times 10^{-9}$ \\
S5 & $3.60 \times 10^{-6}$ & $1.11 \times 10^{-9}$ & $1.12 \times 10^{-9}$ & $2.22 \times 10^{-9}$ \\
S6 & $5.10 \times 10^{-6}$ & $1.57 \times 10^{-9}$ & $1.58 \times 10^{-9}$ & $3.15 \times 10^{-9}$ \\
S7 & $5.70 \times 10^{-6}$ & $1.75 \times 10^{-9}$ & $1.77 \times 10^{-9}$ & $3.52 \times 10^{-9}$ \\
S8 & $5.40 \times 10^{-6}$ & $1.66 \times 10^{-9}$ & $1.67 \times 10^{-9}$ & $3.33 \times 10^{-9}$ \\
\hline
\end{tabular}




\section{CONCLUSIONS}

This paper constructs the evaluation model of the civil and military aviation collision risk, deduces the corresponding calculation method, and uses this method to analyze the collision risk of the "8" motor and the civil aviation route. The concrete conclusion is as follows:

1) In order to reduce the risk of collision, "8" motor training starting point should be set close to the side of the civil aviation route, and began to be circled to the route side.

2 ) The direction of the flight should be decided by the direction of the flight of civil aviation aircraft based on the height of the aircraft. The direction of military aircraft closest to the route is the same with the civil aviation aircraft.

3 ) In accordance with the most dangerous conditions, the interval of $7 \mathrm{~km}$ between the ideal hover trajectory and airspace boundary meets the requirements of the target safety level. Therefore, security interval of the trajectory and the airspace boundary in the route side should be based on the actual situation in the process of designating hover training airspace.

4) The improved Event model was used to evaluate the collision risk between the military aircraft in the circling training airspace and the civil aviation aircraft in the croute. The evaluation results show that the current safety interval distance of 10 $\mathrm{km}$ in the military and civil aviation cannot guarantee that the collision probability between civil aviation and civil aviation satisfies the target safety level. By setting the collision risk under different security redundancy, the suggestions and suggestions on the training of airspace hover training airspace are given.

\section{ACKNOWLEDGMENT}

In this paper, the research was sponsored by the Key Technique of Unmanned Aerial Vehicle Airspace Safety (KGKT05140501)

\section{REFERENCES}

[1] REICH P G. Analysis of long range air traffic systems: separation standards III[J]. Journal of the Institute of Navigation, 1966, 19(3): 331-347.

[2] BROOKER P. Lateral collision risk in air traffic systems: a "Post-Reich” event model[J]. Journal of Navigation, 2003, 56(3): 399-409.

[3] XU Xiaohao, LI Dongbin, LI Xiong. Research on safety assessment of flight separation[J]. Acta Aeronautica et Astronautica Sinica, 2008, 29(6): 1411-1418.

[4] XU Xiaohao, WANG Zhenyu, ZHAO Hongsheng. Improved lateral collision risk model based on Event[J]. Journal of Civil Aviation University of China, 2008, 26(3): 1-4.

[5] ZHANG Zhaoning, LIU Jimin. Assessment of collision risk of vertical separation based on CNS performance[J]. Journal of Civil Aviation University of China, 2010, 28(1): 5-8, 37.

[6] MENG Xiangwei, ZHANG Ping, WANG Ying. Aircraft collision risk assessment at intersecting air routes[J]. Journal of Beijing University of Aeronautics and Astronautics, 2010, 36(9): 1021-1025.

[7] WANG Lili, ZHANG Zhaoning, LIU Jimin. Study of CNS performance environment based on safe separation[J]. Mathematics in Practice and Theory, 2011, 41(19): 23-29.

[8] ZHANG Xiaoyan, ZHU Daiwu, YANG Changqi. Safety assessment of RNP4 Chengdu-Lasa parallel routes[J]. Science Technology and Engineering, 2014, 14(29): 308-311.

[9] CAO Xingwu, ZHANG Zhaoning. Cross route collision risk assessment based on improved Event model[J]. Journal of Civil Aviation University of China, 3 2015,

[10] 